\title{
Phonon transmission through defects in carbon nanotubes from first principles
}

\author{
N. Mingo, ${ }^{1,2}$ D. A. Stewart, ${ }^{3}$ D. A. Broido, ${ }^{4}$ and D. Srivastava ${ }^{2}$ \\ ${ }^{1}$ CEA-Grenoble, 17 rue des Martyrs, 38000 Grenoble, France \\ ${ }^{2}$ University of California at Santa Cruz, Santa Cruz, California 95064, USA \\ ${ }^{3}$ Cornell Nanoscale Facility, Cornell University, Ithaca, New York 14853, USA \\ ${ }^{4}$ Department of Physics, Boston College, Chestnut Hill, Massachusetts 02467, USA
}

(Received 8 June 2007; revised manuscript received 14 September 2007; published 30 January 2008)

\begin{abstract}
We compute the effect of different isolated defects on the phonon transmission through carbon nanotubes, using an $a b$ initio density functional approach. The problem of translational and rotational invariance fulfillment in the nonperiodic system is solved via a Lagrange-multiplier symmetrization technique. The need for an $a b$ initio approach is illustrated for the case of phonon transmission through a nitrogen substitutional impurity, for which no reliable empirical interatomic potentials exist. This opens an avenue for the accurate parameterfree study of phonon transport through general systems with arbitrary composition and structure, without any need for semiempirical potential descriptions.
\end{abstract}

DOI: 10.1103/PhysRevB.77.033418

PACS number(s): 68.65.-k, 63.22. $-\mathrm{m}, 65.80 .+\mathrm{n}, 71.15 . \mathrm{Mb}$

Understanding phonon conduction at the nanoscale is fundamental to addressing power dissipation in nanodevices, ${ }^{1}$ which is one of the important problems faced by current electronic miniaturization. ${ }^{2}$ In recent years, it has been possible to measure thermal conduction through a number of individual nanoscale systems, such as nanowires, nanotubes, nanobelts, and molecular junctions, among others. ${ }^{3-10}$ In many cases, semiempirical theoretical computations of phonon conduction can yield good agreement with experimental results. ${ }^{11-14}$ However, first-principles (or $a b$ initio) studies of phonon conduction are extremely scarce, ${ }^{15}$ and no firstprinciples calculation of phonon flow in nanoscale systems appears to have been published. In this Brief Report we develop the techniques necessary to compute phonon transmission probabilities through inhomogeneous systems, fully from first principles, without any adjustable parameters. We illustrate its use by computing the phonon transmission through single defects in single walled carbon nanotubes (SWCNT's), and when possible, we compare the results with those from parametrized interatomic potentials. We concretely prove that the effect of nitrogen substitutional impurities on phonon conduction through SWCNT's is much weaker than that of lattice defects. This fact can only be computationally assessed via a first-principles calculation, due to the lack of reliable interatomic potentials describing the N-C bond. Thus, the nitrogen example highlights the importance of using an $a b$ initio approach in phonon transport calculations.

The goal of many nanoscale thermal transport experiments is to determine the heat flow $J_{Q}$ between two "reservoirs" at different temperatures joined by an object of nanoscale dimensions. When the temperature difference is small one obtains the thermal conductance of the nanojunction, $\sigma=J_{Q} / \Delta T$. In electrical insulators this magnitude directly depends on the way phonons propagate through the nanostructure.

The phonon dynamics, in an atomic level representation, are described by a many body Hamiltonian $\hat{H}=\frac{1}{2} \sum_{i j} K_{i j} \hat{\varphi}_{i} \hat{\varphi}_{j}$ $+\frac{1}{2} \sum_{i} M_{i} \hat{\dot{\varphi}}_{i} \hat{\dot{\varphi}}_{i}+\mathcal{O}_{a n h}$. The first two terms are single particle terms governing the elastic processes in the lattice. The rest contains the anharmonic terms. $\hat{\varphi}_{i}$ is the Heisenberg displacement operator for the $i$ th atomic degree of freedom. The force constants appearing in the Hamiltonian are defined as $K_{i j} \equiv \partial^{2} E / \partial u_{i} \partial u_{j}$, where $E$ is the total energy, and the $u$ 's are the atomic coordinates. The mass of the atom for the $i$ th degree of freedom is given by $M_{i}$.

Under steady state with a finite temperature difference, an energy current flows between the reservoirs. This current can be calculated in the general anharmonic case. ${ }^{16}$ In carbon nanotubes, it has been demonstrated that anharmonic scattering does not affect phonon transport noticeably below room temperature for samples below micrometer length. $5,8,14,17$ With shorter samples, the temperature at which anharmonicity becomes noticeable is even higher (almost $1000 \mathrm{~K}$ for a $100 \mathrm{~nm}$ long sample). ${ }^{14}$ If the dominant scattering mechanism is elastic, as due to impurities or defects, the equations can be simplified to those of the harmonic problem as follows:

$$
\sigma=\frac{1}{k_{B} T^{2}} \int_{0}^{\infty}(\hbar \omega)^{2} \frac{e^{\hbar \omega / k_{B} T}}{\left(1-e^{\hbar \omega / k_{B} T}\right)^{2}} \mathcal{T}(\omega) d \omega / 2 \pi .
$$

The properties of the system under consideration are contained in the phonon transmission function, $\mathcal{T}(\omega)$, which is calculated from the force constants and the phonon retarded Green's functions. ${ }^{18}$

If one could compute the force constants from first principles, the problem of parameter-free phonon transmission would be solved. However, there is a difficulty: the force constants, $K_{i j}$, must be invariant with respect to rigid translations and rotations of the system; but in general, ab initio force constants do not satisfy these invariances. There are six such conditions, expressed as $\sum_{j} K_{i j} R_{j}^{n}=0$, where $R_{j}^{n}$ is the displacement of the $j$ th degree of freedom upon one of the translations or rotations, labeled by $n=1, \ldots, 6$. Physically meaningful parametrized interatomic potentials automatically satisfy the symmetry conditions. ${ }^{19}$ In contrast, for $a b$ initio force constants there is no built-in cutoff interatomic distance beyond which the interactions vanish. Although they become very small beyond a certain distance, they are 
still finite. Thus, for an extended system, the translation and/or rotation invariance conditions would in principle require an extremely large number of neighbors to be included. An efficient way to deal with this problem in periodic systems is the cumulant force constant method of Parlinski et al. ${ }^{20}$ which does not require any truncation of the interactions. This approach yielded accurate results for the phonon dispersions of carbon nanotubes. ${ }^{21}$

When computing phonon propagation through nonperiodic systems such as single interfaces, however, most atoms are inequivalent, and one needs to impose a cutoff at a finite number of nearest neighbors in order for the calculation to be manageable by Green's function techniques. Imposing this cutoff does not affect most of the vibrational spectrum noticeably, since the neglected interactions are very small. However, now the symmetry invariances are not satisfied, and therefore the calculated phonons in the lowest frequency range behave unphysically. For example, the phonon transmission through a single defect on a nanotube would unphysically decrease below 4 when $\omega \rightarrow 0$, whereas it can be shown that for any infinitely long nanotube or nanowire, with a finite defective fraction in an otherwise pristine periodic system, the zero frequency transmission must always be 4 . This is because there are always four eigenmodes with zero frequency, corresponding to the three rigid translations, and the rotation around the wire's axis. (This rule does not apply in other systems: for example, if a junction links two different systems, with different structure or different mass, in which case the transmission is lower than 4 at low frequency.) To avoid these unphysical behaviors, it is essential to symmetrize the force constants of the nonperiodic system so that the translational and rotational invariances are satisfied.

We now introduce an efficient symmetrization procedure, applicable to nonperiodic systems. The symmetrized force constants must be such that they differ from the original set by as little as possible. Let us denote the symmetrized constants by $\widetilde{K}$, so that

$$
\tilde{K}_{i j} \equiv K_{i j}+D_{i j},
$$

where the $D$ 's denote the differences between the two sets. A measure of how different the two sets are is given by a scalar error function, $f$. There are different ways of defining $f$. In this work we opted for a weighted form,

$$
f \equiv \sum_{i j} D_{i j}^{2} / K_{i j}^{2} .
$$

The symmetry conditions to be satisfied by the new set are

$$
\phi_{i}^{n} \equiv \sum_{j}\left(D_{i j}+K_{i j}\right) R_{j}^{n}=0,
$$

with $n=1, \ldots, 6$. This can be also written as

$$
\begin{gathered}
\sum_{j} D_{i j} R_{j}^{n}=a_{i}^{n}, \\
a_{i}^{n} \equiv-\sum_{j} K_{i j} R_{j}^{n} .
\end{gathered}
$$

The quantities $a_{i}^{n}$ are known. Now we need to determine the unknown quantities $D_{i j}$ that satisfy Eq. (5), and which also yield the smallest possible $f$ Eq. (3). Such a problem is solved by the method of Lagrange multipliers. The standard multiplier equations are $^{22}$

$$
\frac{\partial f}{\partial D_{i j}}+\frac{\partial f}{\partial D_{j i}}-\sum_{n}\left(\lambda_{i}^{n} \frac{\partial \phi_{i}^{n}}{\partial D_{i j}}+\lambda_{j}^{n} \frac{\partial \phi_{j}^{n}}{\partial D_{j i}}\right)=0,
$$

where the multipliers $\lambda$ are not known, and we have used the fact that $D_{i j}=D_{j i}$. Generally, it is not necessary to explicitly determine the values of the multipliers. However, we found it convenient to solve for the $\lambda$ 's first, and evaluate the $D$ 's from them.

Working out the terms in the previous equation yields the $D$ 's in terms of the multipliers as follows:

$$
D_{i j}=(1 / 4) K_{i j}^{2} \sum_{m}\left(\lambda_{i}^{m} R_{j}^{m}+\lambda_{j}^{m} R_{i}^{m}\right) .
$$

Substituting this into the symmetry conditions, Eq. (5), yields

$$
(1 / 4) \sum_{j} \sum_{m} K_{i j}^{2}\left\{R_{j}^{m} R_{j}^{n} \lambda_{i}^{m}+R_{i}^{m} R_{j}^{n} \lambda_{j}^{m}\right\}=a_{i}^{n} .
$$

The equation can be rearranged in a more symmetric form as

$$
\begin{gathered}
\sum_{j} \sum_{m} B_{i, j}^{n, m} \lambda_{j}^{m}=a_{i}^{n}, \\
B_{i, j}^{n, m} \equiv \frac{1}{4}\left(\sum_{k} K_{i k}^{2} R_{k}^{m} R_{k}^{n}\right) \delta_{i j}+\frac{1}{4} R_{i}^{m} R_{j}^{n} K_{i j}^{2} .
\end{gathered}
$$

The $\lambda$ 's are obtained by solving Eq. (10), and the $D$ 's are directly obtained from them by Eq. (8). Then, the symmetrized force constants are immediately known via Eq. (2).

As an alternative to the method presented, it might in principle be possible to symmetrize force constants in nonperiodic systems using the technique of Refs. 23 and 24. This latter approach is not based on Lagrange multipliers, but uses geometrical distance minimization arguments in a high dimensional space. The elements of this space in Ref. 24 are very large vector arrays, which require a much larger memory allocation than the matrix based method we have presented.

Although atomistic investigations of phonon transmission through nonperiodic structures have been previously reported, ${ }^{18,25-30,32}$ all previous work relied on empirical potentials. We now demonstrate our first-principles approach to describe phonon transport through isolated defects on a $(7,0)$ SWCNT. To obtain the (unsymmetrized) force constants, we employed the atomic orbital basis pseudopotential density functional approach of Ref. 33. Other first-principles methods can also be used to obtain force constants. ${ }^{21,34}$ The symmetrization technique does not change, regardless of the $a b$ initio method employed. For the present calculations, a double zeta basis set was used. We assumed the local density approximation. A grid cutoff of 210 Ry was used and Brillouin zone sampling was done using a $[1 \times 1 \times 15]$ Monkhorst-Pack grid. Large unit cells were set up comprising 140 atoms, and it was verified that the results did not 


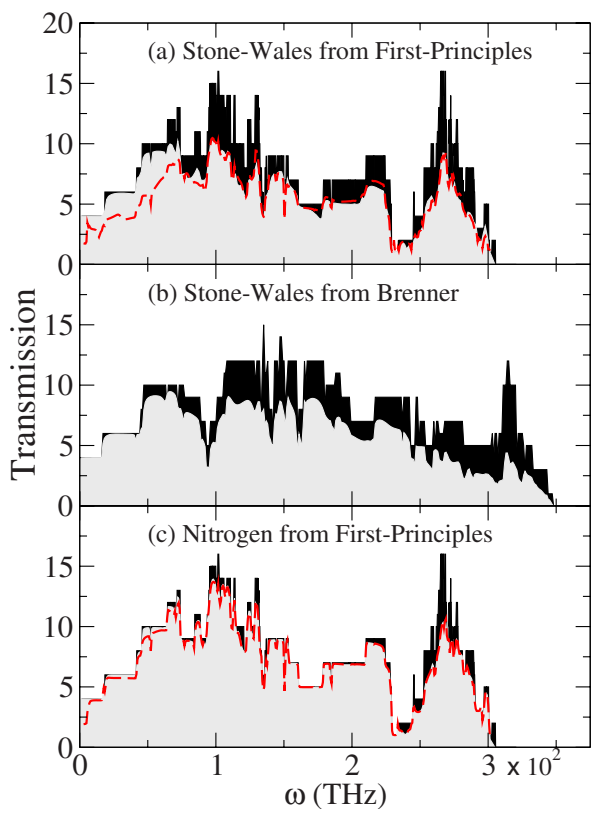

FIG. 1. (Color online) Gray shading shows phonon transmission for (a) Stone-Wales defect from first principles, (b) Stone-Wales defect from Brenner potential, and (c) nitrogen substitutional impurity from first principles, on a $(7,0)$ SWCNT. Black shading shows phonon transmission for the pristine nanotube. Dashed red lines show the (unphysical) results obtained using unsymmetrized force constants.

appreciably change with size. No changes were observed in the symmetrized results when using cutoff radii longer than five nearest neighbor rings.

We investigated two different examples of phonon transmission through an individual defect, in an otherwise pristine carbon nanotube. The first example is a structural defect of the Stone-Wales type, ${ }^{35}$ consisting in the rotation of one of the $\mathrm{C}-\mathrm{C}$ bonds by $90^{\circ}$, to form a heptagon-pentagon structure. The resulting transmission function, $\mathcal{T}(\omega)$, is considerably decreased with respect to the pristine nanotube case [Fig. 1(a)]. This result can be compared with the one obtained using the Brenner interatomic empirical potential [Fig. 1(b)]. Qualitatively, the two cases show similar degrees of reduction in the transmission when the defect is present. However, there are important quantitative differences in the distribution of peaks, and the extension of the spectrum. The Brenner potential overestimates the frequency extension of the spectrum with respect to the experimental one by about $15 \%$. Transmission curves similar to Fig. 1(b) were shown in Ref. 30 for armchair nanotubes at low frequency, using a Brenner potential.

The next example illustrates the effect of a nitrogen substitutional impurity on the SWCNT thermal conductance. Because of the lack of standard empirical force fields for the $\mathrm{C}-\mathrm{N}$ interaction, it would be difficult to assess this effect without a first-principles calculation. Nitrogen impurities on carbon nanotubes can be magnetic in certain configurations, but the most stable, $s p^{2}$ substitutional configuration studied here does not display any magnetism. ${ }^{31}$ Figure $1(\mathrm{c})$ shows that the transmission is affected to a much lesser extent than
TABLE I. Calculated thermal conductance, $\sigma$, at different temperatures, for the pristine, nitrogen substitutional impurity, and Stone-Wales defect cases, on a $(7,0)$ SWCNT. Also shown are results using the parametrized Brenner potential.

\begin{tabular}{lcccccc}
\hline \hline & \multicolumn{2}{c}{$\sigma(\mathrm{nW} / \mathrm{K})($ (ab initio $)$} & & \multicolumn{2}{c}{$\sigma(\mathrm{nW} / \mathrm{K})($ Brenner $)$} \\
\cline { 2 - 4 } \cline { 6 - 7 }$T(\mathrm{~K})$ & Pristine & Nitrogen & S.W. & & Pristine & S.W. \\
\hline 100 & 0.577 & 0.553 & 0.524 & & 0.564 & 0.540 \\
300 & 2.26 & 2.18 & 1.85 & & 2.28 & 1.89 \\
1000 & 4.51 & 4.23 & 3.45 & & 5.15 & 3.81 \\
\hline \hline
\end{tabular}

in the Stone-Wales case. This is because the force constants of the impurity system are very similar to those of the pure carbon system. The effect on the transmission is mostly due to the mass difference between the $\mathrm{N}$ and $\mathrm{C}$ atoms. Most of the reduction takes place at the higher frequency part of the spectrum, so the main effect on thermal conductance will be noticeable at high temperature. The high temperature limit of the thermal conductance is proportional to the area under the transmission curve. The nitrogen impurity only decreases the thermal conductance by $6 \%$ at most. The first-principles thermal conductance, $\sigma$, is calculated using Eq. (1) at 100, 300, and $1000 \mathrm{~K}$ for the pristine case, the nitrogen impurity, and the Stone-Wales defect. Values for $\sigma$ are given in Table I and compared to the results from the Brenner potential. The importance of symmetrization is clear from the dashed lines in Fig. 1, calculated with the unsymmetrized force constants. In those cases, the zero frequency transmission is unphysically lower than 4.

All the transmissions presented correspond to nanotubes infinitely extended on both sides around the defect. Some comments are in order regarding the influence of real contacts. It is a fact that for weak and long enough contacts (adiabatic contacts) the harmonic transmission coincides with that of an infinitely extended nanotube. ${ }^{36}$ An abrupt contact, however, can affect the transmission. We evaluated the effect of an abrupt contact as a function of the contacted length, using an approximate contact self-energy to mimic a surrounding amorphous carbon. ${ }^{18}$ Contact lengths of $400 \mathrm{~nm}$ are sufficient to saturate the transmission to the infinitely long contact value. The fact that the junction is abrupt decreased the room temperature conductance by about $10 \%$ with respect to the infinite nanotube case. Weaker, longer contacts allow for a transmission closer to the infinite nanotube case (i.e., the adiabatic contact case). A full investigation of this issue falls beyond the scope of this Brief Report and will be presented elsewhere.

The approach exemplified here is generally applicable to any nonperiodic system. It can thus be useful for the firstprinciples study of thermal transport through interfaces, disordered systems, ${ }^{37}$ or amorphous materials, avoiding the need to develop any prior force fields. Anharmonic effects could also be included using the formalism developed in Refs. 16 and 38. One could ask whether anharmonicity may be more pronounced in the substitutional impurity case, due to vibrations concentrating near the impurity. To address this we included an anharmonic self energy over one unit cell of 
the system, obtaining no significant differences between the anharmonically induced decrease of the conductance in the $\mathrm{N}$ substituted and the pristine cases at $1000 \mathrm{~K}$. This is due to the fact that the mass of $\mathrm{N}$ and $\mathrm{C}$ are not very different, and also the energy derivatives with respect to atomic displacements are almost the same in the two cases. The study of anharmonicity over realistic lengths requires the development of efficient algorithms and approximations for the anharmonic self energies, which are beyond the scope of the present Brief Report.

To conclude, we implemented a first-principles method to investigate the transmission of phonons across atomically defined nonperiodic systems. We were able to study phonon transmission through a single nitrogen substitutional impurity in a carbon nanotube, for which no adequate parametrized force fields exist. Such a calculation would not be feasible without a first-principles approach. We also investigated phonon transmission through Stone-Wales defects in carbon nanotubes, and compared the first-principles results with those using the Brenner potential. It was found that the effect of the nitrogen impurity is rather minor as compared with that produced by structural defects of the Stone-Wales type. We also described the problem of fulfilling translational and rotational invariances when using first principles, and we developed a Lagrange-multiplier approach to solve it. The method shown here should open an avenue for the calculation of phonon transport across interfaces and heterogeneous systems, without the need to use previously developed interatomic potentials.

We thank M. Lazzeri for providing Ref. 24, and L. FoaTorres for discussions. Computations were run on the Intel Computing Cluster at the Cornell Nanoscale Facility, a member of the National Nanotechnology Infrastructure Network supported by NSF. This project was supported in part by NSF Grants No. 0651310, No. 0651427, and No. 0651381.
${ }^{1}$ D. G. Cahill, W. K. Ford, K. E. Goodson, G. D. Mahan, A. Majumdar, H. J. Maris, R. Merlin, and S. R. Phillpot, J. Appl. Phys. 93, 793 (2003).

${ }^{2}$ ITRS 2005 (http://public.itrs.net/).

${ }^{3}$ K. Schwab, E. A. Henriksen, J. M. Worlock, and M. L. Roukes, Nature (London) 404, 974 (2000).

${ }^{4}$ J. Hone et al., Appl. Phys. Lett. 77, 31 (2000).

${ }^{5}$ P. Kim, L. Shi, A. Majumdar, and P. L. McEuen, Phys. Rev. Lett. 87, 215502 (2001).

${ }^{6}$ L. Shi, D. Li, C. Yu, W. Jang, D. Kim, Z. Yao, P. Kim, and A. Majumdar, J. Heat Transfer 125, 881 (2003).

${ }^{7}$ D. Li, Y. Wu, R. Fan, P. Yang, and A. Majumdar, Appl. Phys. Lett. 83, 3186 (2003).

${ }^{8}$ H.-Y. Chiu, V. V. Deshpande, H. W. Ch. Postma, C. N. Lau, C. Miko, L. Forro, and M. Bockrath, Phys. Rev. Lett. 95, 226101 (2005).

${ }^{9}$ E. Pop, D. Mann, Q. Wang, K. E. Goodson, and H. Dai, Nano Lett. 6, 96 (2006).

${ }^{10}$ P. Reddy, S.-Y. Jang, R. A. Segalman, and A. Majumdar, Science 315, 1568 (2007).

${ }^{11}$ N. Mingo, Phys. Rev. B 68, 113308 (2003).

${ }^{12}$ L. Shi, Q. Hao, Ch. Yu, N. Mingo, X. Kong, and Z. L. Wang, Appl. Phys. Lett. 84, 2638 (2004).

${ }^{13}$ N. Mingo and D. A. Broido, Phys. Rev. Lett. 95, 096105 (2005).

${ }^{14}$ N. Mingo and D. A. Broido, Nano Lett. 5, 1221 (2005).

${ }^{15}$ D. A. Broido, M. Malorny, G. Birner, Natalio Mingo, and D. A. Stewart, Appl. Phys. Lett. 91, 231922 (2007).

${ }^{16}$ N. Mingo, Phys. Rev. B 74, 125402 (2006).

${ }^{17}$ C. Yu, L. Shi, Z. Yao, D. Li, and A. Majumdar, Nano Lett. 5, 1842 (2005).

${ }^{18}$ N. Mingo and Liu Yang, Phys. Rev. B 68, 245406 (2003); 70, 249901(E) (2004).

${ }^{19}$ G. D. Mahan and G. S. Jeon, Phys. Rev. B 70, 075405 (2004).

${ }^{20}$ K. Parlinski, Z.-Q. Li, and Y. Kawazoe, Phys. Rev. Lett. 78, 4063 (1997).

${ }^{21}$ L. H. Ye, B. G. Liu, D. S. Wang, and R. Han, Phys. Rev. B 69, 235409 (2004).
${ }^{22}$ G. B. Arfken, Mathematical Methods for Physicists (Academic, New York, 1985).

${ }^{23}$ N. Mounet and N. Marzari, Phys. Rev. B 71, 205214 (2005); in Functional Carbon Nanotubes, edited by D. L. Carroll, B. Weisman, S. Roth, and A. Rubio, MRS Symposia Proceedings No. 858E (Materials Research Society, Warrendale, PA, 2005), p. HH3.34.

${ }^{24}$ N. Mounet, Masters thesis, M.I.T., 2005.

${ }^{25}$ D. A. Young and H. J. Maris, Phys. Rev. B 40, 3685 (1989).

${ }^{26}$ S. Pettersson and G. D. Mahan, Phys. Rev. B 42, 7386 (1990).

${ }^{27}$ G. Fagas, A. G. Kozorezov, C. J. Lambert, J. K. Wigmore, A. Peacock, A. Poelaert, and R. den Hartog, Phys. Rev. B 60, 6459 (1999).

${ }^{28}$ P. K. Schelling, S. R. Phillpot, and P. Keblinski, Appl. Phys. Lett. 80, 2484 (2002).

${ }^{29}$ J. Wang and J. S. Wang, Phys. Rev. B 74, 054303 (2006).

${ }^{30} \mathrm{~T}$. Yamamoto and K. Watanabe, Phys. Rev. Lett. 96, 255503 (2006).

${ }^{31}$ Y. Ma, A. S. Foster, A. V. Krasheninnikov, and R. M. Nieminen, Phys. Rev. B 72, 205416 (2005).

${ }^{32}$ W. Zhang, T. S. Fisher, and N. Mingo, J. Heat Transfer 129, 483 (2007).

${ }^{33}$ J. Soler, E. Artacho, J. D. Gale, A. Garcia, J. Junquera, P. Ordejon, and D. Sanchez-Portal, J. Phys.: Condens. Matter 14, 2745 (2002); D. Sanchez-Portal, E. Artacho, J. M. Soler, A. Rubio, and P. Ordejon, Phys. Rev. B 59, 12678 (1999).

${ }^{34}$ O. Dubay and G. Kresse, Phys. Rev. B 67, 035401 (2003); 69, 089906(E) (2004).

${ }^{35}$ A. J. Stone and D. J. Wales, Chem. Phys. Lett. 128, 501 (1986).

${ }^{36}$ L. G. C. Rego and G. Kirczenow, Phys. Rev. Lett. 81, 232 (1998).

${ }^{37}$ S. Maruyama, Y. Igarashi, Y. Taniguchi, and J. Shiomi, J. Therm. Sci. Technol. 1, 138 (2006); N. Kondo, T. Yamamoto, and K. Watanabe, e-J. Surf. Sci. Nanotechnol. 4, 239 (2006).

${ }^{38}$ J.-S. Wang, J. Wang, and N. Zeng, Phys. Rev. B 74, 033408 (2006). 